\title{
Modelling vacancy microvoid formation in dislocation-free silicon single crystals
}

\author{
V.I. Talanin, I.E. Talanin, S.A. Koryagin, M.Yu. Semikina \\ University of State \& Municipal Government \\ 70B, Zhukovskii str., 69002 Zaporozhye, Kyiv, Ukraine
}

\begin{abstract}
An alternative mathematical model of vacancy microvoid formation in dislocation-free silicon single crystals was represented. The analysis of conditions of microvoid nucleation inside the bulk of crystals during cooling after their growth was carried out. The possibility of formation of a quasi-stationary microvoid profile in largescale crystals within the temperature range $1130 \ldots 1070^{\circ} \mathrm{C}$ has been shown.
\end{abstract}

Keywords: point defect, grown-in microdefect, vacancy microvoid, CZ-Si, FZ-Si.

Manuscript received 10.10.06; accepted for publication 23.10.06.

\section{Introduction}

In dislocation-free silicon single crystals, the formed various point defect agglomerates (i.e. grown-in microdefects) sufficiently influence on both electrophysical, mechanical properties of crystals and characteristics of integrated chips. It is necessary to develop a theoretical model of grown-in microdefect formation that is adequate to experimental results of a research of a dislocation-free silicon single crystal microdefect structure. This is an actual task, it allows to solve the problem of controlling the point defect ensemble.

At the moment, the model of point defect dynamics is generally accepted [1,2]. This model describes convection, recombination, diffusion of intrinsic point defects and, for all its modifications, the Voronkov model of grown-in microdefect formation [3] is basic. The heart of the Voronkov model is a recognition of a critical role of "fast recombination" process of intrinsic point defects close to a crystallization front. Depending on temperature growth conditions, the model of point defect dynamics assumes the formation of interstitial dislocation loops and vacancy microvoids during crystal cooling in the narrow temperature interval below $1200{ }^{\circ} \mathrm{C}$ in various regions of a crystal. Furthermore, it is supposed that the formation of all types of microdefects is of homogeneous nature, and the process of interaction of intrinsic point defects with impurity atoms is ignored [1]. In the model of point defect dynamics, it is implicitly supposed that, depending on the ratio $V / G(V$ is the growth rate of the crystal, $G$ - axial temperature gradient), the defects that were formed within the temperature range $1420 \ldots 1200{ }^{\circ} \mathrm{C}$ are either small vacancy microvoids or small interstitial dislocation loops [3].
As opposed to the model of point defect dynamics, basing on a great body of experimental researches, we have developed the qualitative heterogeneous mechanism of formation and transformation of grown-in microdefects $[4,5]$. It was shown that the defect formation process was controlled by diffusion of point defects in the temperature gradient field. It was caused by availability of an entropy recombinational barrier that blocks the recombination of intrinsic point defects at high temperatures in the course of crystal growth [4]. Therefore, dissociation of the oversaturated solid solution of point defects goes in parallel in two directions: vacancy and interstitial. At the temperatures close to the crystallization front, the dissociation of oversaturated solid solutions of impurities takes place, and in the course of cooling the crystal $\left(T<1200^{\circ} \mathrm{C}\right)$ the dissociation of those of intrinsic point defects (vacancies and self-interstitials) occurs. The absence of a theoretical (mathematical) model for defect formation within the temperature ranges both $1420 \ldots 1200{ }^{\circ} \mathrm{C}$ and $1200 \ldots 900{ }^{\circ} \mathrm{C}$ is currently disadvantage of the heterogeneous mechanism.

In this research, developed was the model of vacancy microvoid formation as a result of condensation of non-equilibrium vacancies during cooling of growing monocrystals at $T<1200{ }^{\circ} \mathrm{C}$. The aim of this paper is the analysis of conditions of microvoid initiation taking into account diffusion of non-equilibrium vacancies deep into the crystal and onto its surface.

\section{The statement of problem}

Vacancy microvoids are formed in large-scale crystals (100 $\mathrm{mm}$ and more). The growth of such crystals is 
characterized by large growth rates, small curvature of crystallization front and axial temperature gradient [6]. The temperature interval of formation and growth of microvoids is $\sim 1130 \ldots 1070{ }^{\circ} \mathrm{C}$, the interval of oxide film growth on microvoid walls is $\sim 1070 \ldots 900{ }^{\circ} \mathrm{C}[6-$ 8]. From these results, we believe that, in the crystal layer with a thickness $\delta$ that corresponds to the conditions of cooling the crystal during its growth from 1130 to $1070{ }^{\circ} \mathrm{C}$ (Fig. 1), an excess of non-equilibrium vacancies $n_{0}$ are produced (Fig. 2).

Binding of oxygen atoms into grown-in microdefects $((\mathrm{I}+\mathrm{V})$-microdefects $)$ with lowering the temperature down to the values $T<1200{ }^{\circ} \mathrm{C}$ can be the reason of initiation of these non-equilibrium vacancies. For these temperature conditions at the first stage, there are both the process of dissociation of oversaturated solution of vacancies and simultaneous process of their diffusion. After the majority of vacancies will be used for microvoid formation, and the concentration of nonequilibrium vacancies becomes rather small, the diffusion process of the remained non-equilibrium vacancies onto the surface and into the bulk of the crystal is accompanied by a coalescence process of microvoids. After completion of this second stage, all the excess vacancies pass onto the crystal surface.

At the first stage, according to [9] the system can be described by the equations

$\frac{\partial n}{\partial t}=D \frac{\partial^{2} n}{\partial x^{2}}-4 \pi N D r n$,

$\frac{\partial r^{2}}{\partial t}=\frac{2 n}{N_{L}} D$

where $n$ is the concentration of non-equilibrium vacancies, $D$ is the diffusivity of vacancies, $r$ is the radius of microvoids, $N_{L}$ is the concentration of vacancies in the microvoid or the reciprocal volume per one vacancy; $N$ is the microvoids concentration.

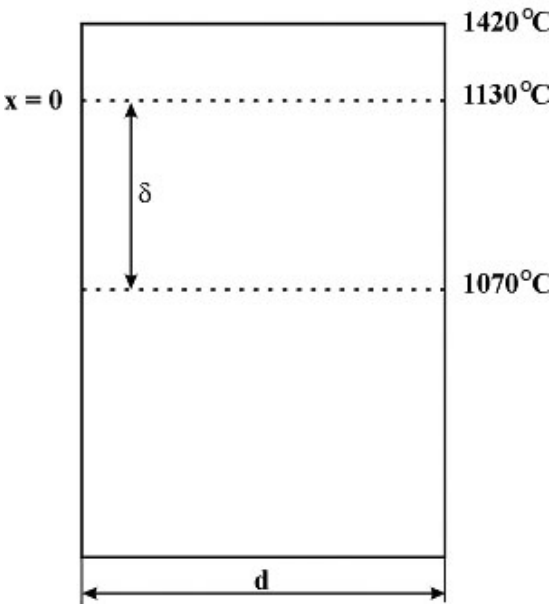

Fig. 1. The scheme of non-equilibrium vacancy formation during crystal cooling in the crystal layer with a thickness $\delta$ ( $d$ is the crystal diameter).
In the right side of Eq. (1), the first member describes diffusion of non-equilibrium vacancies from the A-surface (where the A-surface is the interface between the volume of crystal formed at $1420<T<$ $1200{ }^{\circ} \mathrm{C}$ and the crystal layer with a thickness $\delta$ ) into the depth of a sample, and the second one excludes the part of vacancies that was used for microvoid formation from a vacancy diffusion flux.

According to Figs 1, 2, the boundary and initial conditions look like:

$\left.n\right|_{x=0}=0,\left.n\right|_{t=0}=\left\{\begin{array}{l}n_{0}, \text { at } x \leq \delta \\ 0, \text { at } x>\delta\end{array},\left.r\right|_{t=0}=0,\left.d\right|_{t=0}=\infty\right.$.

The condition $\left.n\right|_{x=0}=0$ assumes that, on the surface (the bulk of crystal formed at $1420<T<1200{ }^{\circ} \mathrm{C}$ plays the role of this surface), very fast absorption of excess vacancies takes place. It follows that the characteristic time of an absorption of excess concentration of vacancies at $x=0$ is much less than all the other characteristic times. The condition $\left.d\right|_{t=0}=\infty$ assumes that in the course of solving the problem we do not take into account a drain of vacancies onto a lateral face of the crystal.

\section{Mathematical model}

Let's consider a solution of the problem for the first stage as is shown in [10]. Let's substitute (2) into (1), integrate with respect to time and enter new variables according to relationships:

$x=\mu \xi, \quad t=v \tau, \quad r^{2}=\lambda q$,

where

$\mu^{6}=\frac{N_{L}}{2 n_{0}} \frac{1}{(8 / 3 \pi N)^{2}}, \quad v=\frac{\mu^{2}}{D}, \quad \lambda=\mu^{-4}(8 / 3 \pi N)^{-2}$.

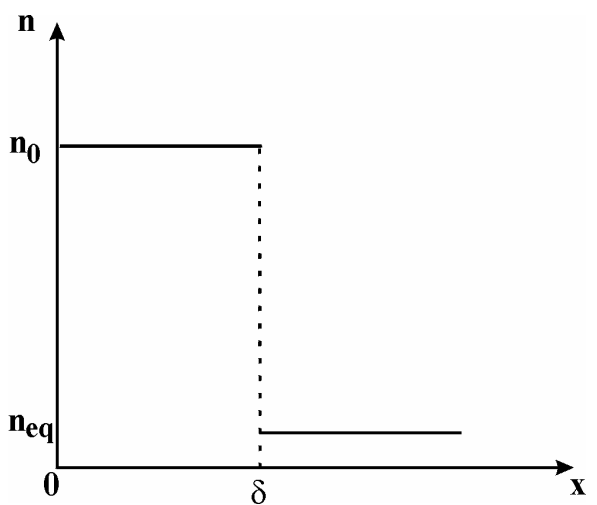

Fig. 2. Profile of vacancies at the initial instant $\left(n_{0}-\right.$ concentration of excess non-equilibrium vacancies; $n_{\mathrm{eq}}-$ equilibrium concentration of vacancies). 
Then the equations (1), (2) take the dimensionless form:

$\frac{\partial q}{\partial \tau}=\frac{\partial^{2} q}{\partial \xi^{2}}-q^{3 / 2}+f(\xi)$

$n=n_{0} \frac{\partial q}{\partial \tau}$

where $f(\xi)=\left\{\begin{array}{l}1, \text { at } \xi \leq \Delta \\ 0, \text { at } \xi>\Delta\end{array}, \quad \Delta=\frac{\delta}{\mu}\right.$

with the boundary and initial conditions

$\left.q\right|_{\tau=0}=0,\left.\quad q\right|_{\xi=0}=0,\left.\quad q\right|_{\xi=\infty}=0$.

The function $f(\xi)$ can be found by substituting the initial conditions into Eq. (4).

Duration of the first stage $\tau_{\mathrm{I}}$ depends on the time of outgoing the majority of excess vacancies into microvoids, which is determined from Eq. (4) with elimination of the diffusion member $\partial^{2} q / \partial \xi^{2}$ that is approximately equal to unity. The restriction $\tau_{\mathrm{I}} \leq \tau_{S}=\Delta^{2}$ is the necessary condition of microvoid formation in the layer with a thickness $\delta$. In accord with this condition, the time of microvoid formation should be no more than the time of vacancy going onto a surface [10].

The characteristic times of the first stage of the process in the dimensional form look like:

$t_{\mathrm{I}}=v \tau_{\mathrm{I}}=\frac{\mu^{2}}{D}$,

$t_{S}=v \tau_{S}=\frac{\delta^{2}}{D}$.

After the completion of the first stage $(\partial q / \partial \tau \approx 0)$, the obtained microvoid profile can be determined from the equation

$\frac{\partial^{2} q}{\partial \xi^{2}}-q^{3 / 2}+f(\xi)=0$

with the boundary conditions $\left.q\right|_{\xi=0}=0,\left.q\right|_{\xi \rightarrow \infty}=0$.

The value $\Delta$ is the parameter that determines the shape of the structure voids. This value is meaningful of the ratio of the characteristic time of going the vacancies onto a surface to the characteristic one of microvoid formation. Solutions of Eq. (7) for three ranges of varying the coordinate $\xi$ look like [10]:

$$
\begin{aligned}
& 0 \leq \xi \leq \xi_{\max }, \quad \int_{0}^{q} \frac{d q}{\sqrt{2\left(\frac{2}{5} q^{5 / 2}-q+q_{\Delta}\right)}}=\xi, \\
& \xi_{\max } \leq \xi \leq \Delta, \quad \xi_{\max }+\int_{q}^{q_{\max }} \frac{d q}{\sqrt{2\left(\frac{2}{5} q^{5 / 2}-q+q_{\Delta}\right)}}=\xi,
\end{aligned}
$$

$\Delta \leq \xi<\infty, \quad q=\frac{400}{\left(\xi+\xi_{0}\right)^{4}}$,

where

$\xi_{\max }=\int_{0}^{q_{\max }} \frac{d q}{\sqrt{2\left(\frac{2}{5} q^{5 / 2}-q+q_{\Delta}\right)}}, \xi_{0}=\left(\frac{400}{q_{\Delta}}\right)^{1 / 4}-\Delta$,

$q_{\Delta}, q_{\max }$ are determined by the equations

$\frac{2}{5} q_{\max }^{5 / 2}-q_{\max }+q_{\Delta}=0$,

$\int_{q_{\Delta}}^{q_{\max }} \frac{d q}{\sqrt{2\left(\frac{2}{5} q^{5 / 2}-q+q_{\Delta}\right)}}=\Delta-\xi_{\max }$

In the limiting cases, we have

$\Delta<<1 \quad q_{\max } \approx q_{\Delta} \approx \frac{\Delta^{2}}{2}, \quad \xi_{1 / 2} \approx 2.2 \Delta+\frac{6.2}{\sqrt{\Delta}}$,

$\Delta>>1 \quad q_{\max } \approx 1, \quad \xi_{1 / 2} \approx \Delta+2.5$

The duration of the second stage of this process (microvoid coalescence) can be evaluated using the expression of the characteristic coalescence time [10]

$t_{\mathrm{II}}=\frac{r^{3} k T}{D \sigma \Omega^{2} n_{\mathrm{eq}}}$,

where $\sigma$ is the surface tension at the interface crystalvacuum.

If in a crystal the process of impurity diffusion takes place, then in this case when the impurity has a chance to pass a distance $\delta$ in a time $t_{\text {imp }}$ that is smaller than $t_{\mathrm{II}}$, it will decorate the vacancy profile. Therefore, the condition of impurity profile formation is the ratio:

$t_{\text {imp }}=\frac{\delta^{2}}{D_{\text {imp }}} \leq t_{\mathrm{II}}$,

where $D_{\text {imp }}$ is the diffusivity of impurity.

\section{Experimental}

The values of parameters necessary for calculations are as follows: $N_{L}=\frac{1}{\Omega}=\frac{1}{2 \cdot 10^{-23} \mathrm{~cm}^{3}}=5 \cdot 10^{22} \mathrm{~cm}^{-3}$

[11]; $N=10^{5} \mathrm{~cm}^{-3} \quad[8] ; \quad G=650 \mathrm{erg} / \mathrm{cm}^{2}$

$D=188.449 \exp (-1.917 / k T) ; \quad k=8.6153 \cdot 10^{-5} \mathrm{eV} / \mathrm{K} ;$

$D_{0}=0.17 \exp (-2.54 / k T) ; n=1.11639 \cdot 10^{27} \exp (-3.9 / k T)$

[11]; $r=100 \mathrm{~nm}=10^{-5} \mathrm{~cm} ; D_{c}=1.9 \exp (-3.1 / k T)$. 


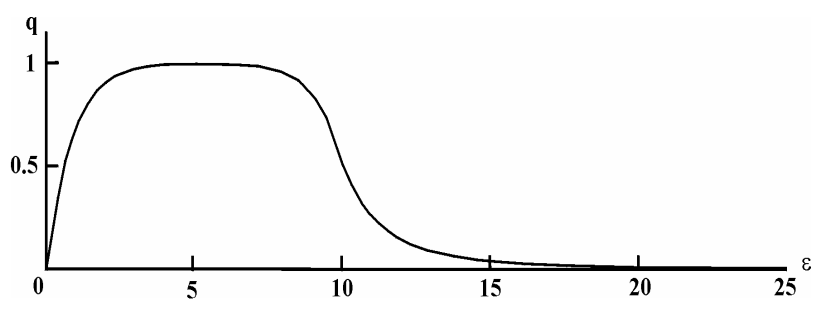

Fig. 3. Dependence of a microvoid radius squared on the distance up to the initial boundary of the temperature interval for microvoid formation (dimensionless, $\Delta=9.767$ ).

The temperature profile along the length of the growing $\mathrm{CZ}-\mathrm{Si}$ and $\mathrm{FZ}-\mathrm{Si}$ crystals is described by the equation [2]: $\frac{1}{T}=\frac{1}{T_{m}}+\frac{1}{T_{m}^{2}} G x$, where $T=1685 \mathrm{~K}$ is the temperature at the crystallization front, $G$ is the axial temperature gradient. Using the standard for majority of CZ-Si crystals value $G=2.5 \mathrm{~K} / \mathrm{mm}$ and vacancy microvoid formation temperature range $1130 \ldots 1070{ }^{\circ} \mathrm{C}$ [6-8], we obtained $\delta \approx 3.75 \mathrm{~cm}$. Then $\mu=0.384 \mathrm{~cm}$ and $\Delta=9.767>1$. For this value of $\Delta$, from Eqs (10)-(12) we determined $\mathrm{q}_{\max }=0.99659, \varepsilon_{\max }=5.284$. The calculation results are shown in Fig. 3.

For the temperature range of vacancy microvoid formation, we evaluated the characteristic times of the first and second stages of the process and concluded that the ratio $t_{\mathrm{I}}<<t_{S}<<t_{\mathrm{II}}$ holds true.

Hence, the unambiguous conclusion follows that, during the crystal growth in a case of creation of excess vacancy concentration in the temperature range of cooling the crystal of $1130 \ldots 1070{ }^{\circ} \mathrm{C}$, the quasistationary microvoid profile is formed. The conditions of vacancy microvoid decoration by background impurities of carbon and oxygen (14) in the temperature interval $1070 \ldots 900{ }^{\circ} \mathrm{C}[6,8]$ are well valid everywhere over the indicated range.

In a statement of the problem, we did not take into account a drain of vacancies onto a lateral face of crystal. The availability of a lateral face limits conditions of vacancy microvoid formation in the cross-section of the crystal to a minimum request $d_{\min } \approx 2 \delta$. Therefore, under a certain temperature growth conditions in commercial single crystals, the vacancy microvoids should be formed since the crystal diameter is more than $80 \mathrm{~mm}$.

\section{Discussion}

The experimental results that were carried out recently by us using the transmission electron microscopy of the FZ-Si and CZ-Si crystals grown in various temperature conditions have shown that the primary oxygen-vacancy and carbon-interstitial agglomerates formed at impurity centres close to a crystallization front are the basis of defect formation process $[4,5]$. The main reason of this process is the availability of a recombinational barrier for intrinsic point defects at high temperatures [12]. Using the impurity atoms to form precipitates results that, depending on the growth conditions when the crystal cooling temperature is lower than $1200{ }^{\circ} \mathrm{C}$, created are the conditions for initiation of a nonequilibrium concentration of vacancies and selfinterstitials of silicon. Therefore, the generation of secondary defects (agglomerates of intrinsic point defects), with which the growth of a new phase is accompanied, is the key feature of dissociation of oversaturated solid solution of point defects.

These experimental results have allowed us to enter a new physical classification of grown-in microdefects, which is based on two types of interaction: the "impurity - intrinsic point defect" (primary grown-in microdefects) and "intrinsic point defect - intrinsic point defect" (secondary grown-in microdefects). Such consideration allows us to enter two defect subsystems (primary and secondary microdefects) and, for each of them, to develop mathematical models that are based on consideration of the phenomena of dissociation of oversaturated solid solutions of point defects within the framework of the dissociative diffusion model.

In this work, as opposite to the Voronkov model, offered was the model of secondary grown-in microdefect formation (vacancy microvoids) that:

- explains the reason of formation of excess nonequilibrium vacancies in the observed temperature range;

- explains the reason of absence of vacancy microvoids in small-scale crystals;

enters the mathematical means that shows the good coordination of the theoretical calculations with the experimental results;

- gives understanding of physics of defect formation process in dislocation-free silicon single crystals;

- $\quad$ is well agreed with the available experimental results.

The theoretical calculations and experimental researches show that the vacancy microvoid formation is caused by initiation of non-equilibrium concentrations of vacancies when cooling the crystal as a result of binding the impurity atoms (in particular, oxygen) in primary grown-in microdefects. In so doing, the primary grownin microdefects (impurity precipitates) are the points of nucleation of vacancy microvoids what is confirmed experimentally. The short-term heat treatment (30 min at $T=1100{ }^{\circ} \mathrm{C}$ ) causes the sharp decrease of sizes and change of microvoid profile which are accompanied by creation of precipitates of significant sizes [8]. It testifies that the vacancies deposit on the oxygen-vacancy and carbon-interstitial agglomerates that are formed close to the crystallization front and grow in the course of crystal cooling. The experimental fact of availability of oxygen in one microvoids and carbon in another microvoids is the confirmation of this contention $[6,7]$. 


\section{Conclusion}

The performed mathematical calculations show that the vacancy microvoid formation in dislocation-free silicon single crystals can be described within the framework of the dissociative diffusion model. In difference from the model of point defect dynamics, the vacancy microvoid formation in the course of crystal cooling within the temperature range $1130 \ldots 1070{ }^{\circ} \mathrm{C}$ correlates with the experimental results on formation of a microdefect structure when cooling within the temperature interval $1420 \ldots 1130{ }^{\circ} \mathrm{C}$. Our consideration of the problem of vacancy microvoid formation confirms that the process of point defect diffusion in the field of a temperature gradient provides the basis for the defect formation in dislocation-free silicon monocrystals during their growth.

\section{Acknowledgements}

This scientific work was made by the budgetary funds of Ministry of Education and Science of Ukraine as the grant of the President of Ukraine.

\section{References}

1. T. Sinno, R.A. Brown, W. von Ammon and E. Dornberger, Point defects dynamics and the oxidation-induced stacking-faults in Czochralskigrown silicon crystals // J. Electrochem. Soc. 145 (1), p. 302-318 (1998).

2. M.S. Kulkarni, V. Voronkov and R. Falster, Quantification of defect dynamics in unsteady-state and steady-state Czochralski growth of monocrystalline silicon // J. Electrochem. Soc. 151 (5), p. G663-G669 (2004).

3. V.V. Voronkov, Mechanism of swirl defects formation in silicon // J. Cryst. Growth 59 (3), p. 625-642 (1982).
4. V.I. Talanin and I.E. Talanin, Mechanism of formation and physical classification of the grown in microdefects in semiconductor silicon // Defect \& Diffusion Forum 230-232 (1), p. 177-198 (2004).

5. V.I. Talanin and I.E. Talanin, Formation of grownin microdefects in dislocation-free silicon monocrystals, In: New research on semiconductors, Ed. T.B. Elliot, p. 35-59. Nova Sci. Publ., New York, 2006.

6. T. Ueki, M. Itsumi, T. Takeda, K. Yoshida, A. Takaoka and S. Nakajima, Shrinkage of grownin defects in Czochralski silicon during thermal annealing in vacuum // Jpn J. Appl. Phys. 37 (7), p. L771-L773 (1998).

7. Y. Yanase, H. Nishihata, T. Ochiai, H. Tsuya, Atomic force microscope observation of the change in shape and subsequent disappearance of "crystaloriginated particles" after hydrogen-atmosphere thermal annealing // Jpn J. Appl. Phys. 37 (1), p. 14 (1998).

8. M. Itsumi, Octahedral void defects in Czochralski silicon // J. Cryst. Growth 237-239 (3), p. 17731778 (2002).

9. V.I. Fistul, V.I. Petrovskii, N.S. Rytova and P.M. Grinshtein, Formation of nearsurface impurity profile // Fizika i Technika Poluprovodnikov 13 (11), p. 1402-1410 (1979) (in Russian).

10. V.I. Fistul and M.I. Sinder // Fizika $i$ Technika Poluprovodnikov 15 (6), p. 1182-1186 (1981) (in Russian).

11. M. Akatsuka, M. Okui, S. Umeno and K. Sueoka, Calculation of size distribution of void defects in CZ silicon // J. Electrochem. Soc. 150 (9), p. G587G590 (2003).

12. V.I. Talanin and I.E. Talanin, Recombination parameters of point defects in dislocation-free silicon single crystals // Functional Materials 13 (1), p. 1-5 (2006). 